\title{
Toward Functional Screening of Cardioactive and Cardiotoxic Drugs with Zebrafish in Vivo Using Pseudodynamic Three-Dimensional Imaging
}

\author{
Kuen-You Lin, ${ }^{\dagger}, \#$ Wei-Tien Chang, ${ }^{\ddagger}, \#$ Yu-Cheng Lai, ${ }^{\dagger}$ and Ian Liau* ${ }^{*}$ \\ ${ }^{\dagger}$ Department of Applied Chemistry and Institute of Molecular Science, National Chiao Tung University, Hsinchu 300, Taiwan \\ ${ }^{\ddagger}$ National Taiwan University Hospital and College of Medicine, Taipei 100, Taiwan
}

Supporting Information

\begin{abstract}
Given the high mortality in patients with cardiovascular diseases and the life-threatening consequences of drugs with unforeseen adverse effects on hearts, a critical evaluation of the pharmacological response of cardiovascular function on model animals is important especially in the early stages of drug development. We report a proof-of-principle study to demonstrate the utility of zebrafish as an analytical platform to predict the cardiac response of new drugs or chemicals on human beings. With pseudodynamic 3D imaging,

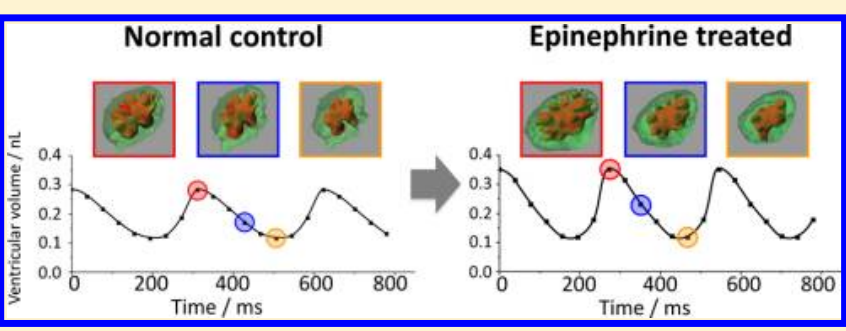
we derive individual parameters that are central to the cardiac function of zebrafish, including the ventricular stroke volume, ejection fraction, cardiac output, heart rate, diastolic filling function, and ventricular mass. We evaluate both inotropic and chronotropic responses of the heart of zebrafish treated with drugs that are commonly prescribed and possess varied known cardiac activities. We reveal deranged cardiac function of a zebrafish model of cardiomyopathy induced with a cardiotoxic drug. The cardiac function of zebrafish exhibits a pharmacological response similar to that of human beings. We compare also cardiac parameters obtained in this work with those derived with conventional $2 \mathrm{D}$ approximation and show that the latter tends to overestimate the cardiac parameters and produces results of greater variation. In view of the growing interest of using zebrafish in both fundamental and translational biomedical research, we envisage that our approach should benefit not only contemporary pharmaceutical development but also exploratory research such as gene, stem cell, or regenerative therapies targeting congenital or acquired heart diseases.
\end{abstract}

$\mathrm{T}^{\mathrm{k}}$ he screening of chemicals for therapeutic effects ${ }^{1-3}$ or testing of new drugs for undesirable toxicity ${ }^{4-6}$ on model animals is an inevitable component of contemporary pharmaceutical development. Given the high mortality in patients with cardiovascular diseases ${ }^{7}$ and the critical consequences of drugs with adverse side effects on hearts (for example, the widely recognized cardiac abnormalities induced by chemotherapeutic agent doxorubicin), ${ }^{8}$ it is of particular importance to evaluate the pharmacological response of the cardiac function of model animals especially in the early stages of drug development. In this regard, the zebrafish (Danio rerio) possesses numerous advantages including convenience of delivery of drugs, rapid development, ease of genetic manipulation, and low cost of maintenance relative to other model animals such as rodents. ${ }^{9,10}$ The underlying function, gene, pathophysiology, and pharmacological response of the cardiovascular system of zebrafish are similar to those of human beings, ${ }^{11,12}$ further making this model organism particularly attractive for the evaluation of the safety and efficacy of drugs that target cardiovascular diseases. ${ }^{4,5}$

A comprehensive assessment of the cardiac response requires an accurate determination of not only the electrical regulation but also mechanical function of a heart. However, screening of drugs for cardiac toxicity or activity using zebrafish models is achieved mostly with cardiac electrophysiological or rhythmic measurements primarily on the electrical aspect of cardiac function; in contrast, pharmacological response of some important mechanical aspects of the cardiac function of zebrafish, such as cardiac output, are much less explored. Cardiac output is defined as the ventricular stroke volume multiplied by the heart rate. Although the heart rate of zebrafish can be readily measured with echocardiography, ${ }^{13}$ electrocardiography, ${ }^{14}$ or digital imaging, ${ }^{4}$ accurate determination of the ventricular stroke volume, a three-dimensional (3D) quantity, of a rapidly beating heart of zebrafish (about 190 beats per minute) remains challenging. The ventricular volume is consequently deduced generally through an approximation, according to which one first obtains two-dimensional (2D) images at a section selected by the user; the $3 \mathrm{D}$ volumetric quantity is then estimated assuming a specific shape (typically an ellipsoid) of the ventricular chamber. ${ }^{15}$ This crude approximation might impose a significant error and, hence, complicate an interpretation of the results. Liebling and co-

Received: November 29, 2013

Accepted: January 23, 2014

Published: January 23, 2014 

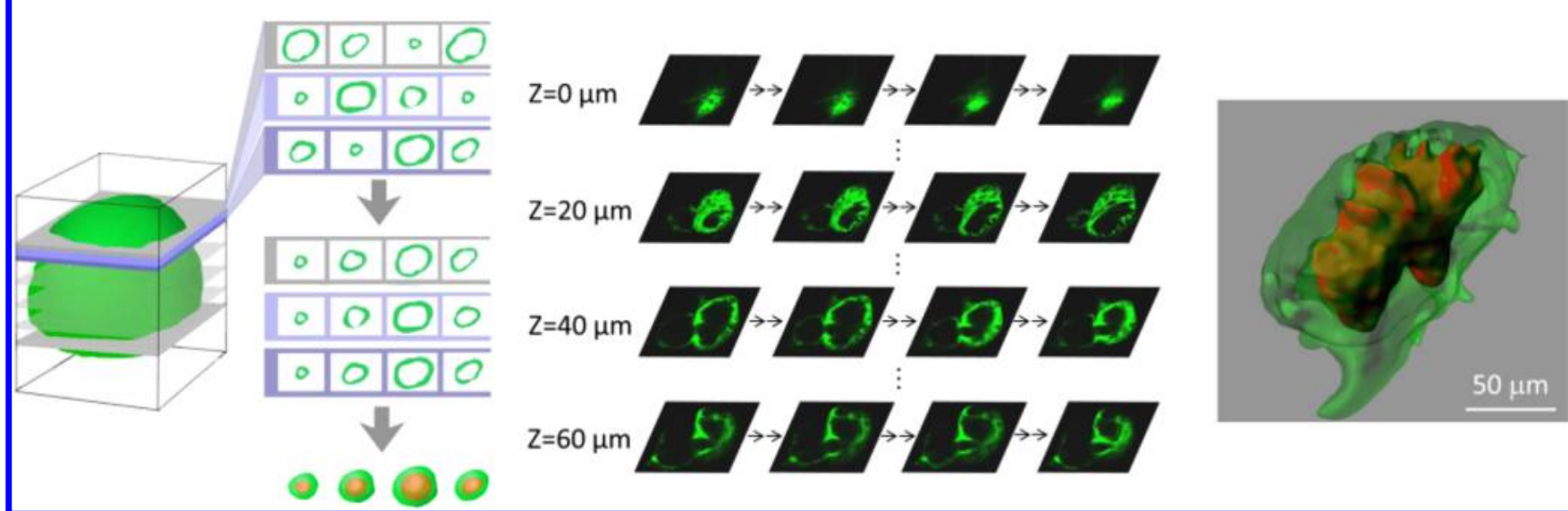

Figure 1. (A) A cartoon illustrating the protocol employed to reconstruct 3D images of a periodically beating heart. (B) Time-lapse confocal fluorescence images of a beating heart of zebrafish obtained at varied focal depths. For clarity, only selected images at four depths are displayed. (C) A representative reconstructed 3D image of the ventricle (green: ventricular muscle; red: ventricular chamber).

workers reported a novel algorithm to reconstruct the dynamic volume of a periodically moving microscopic object with rapid confocal imaging ${ }^{16}$ and characterized the structural and functional development of the heart of zebrafish embryos. ${ }^{17,18}$ Despite unprecedented success in the revelation of the structure-function interplay of an embryonic heart, no application of this approach as a reliable platform for pharmaceutical screening has yet been explicitly demonstrated. Furthermore, particular mechanical and structural parameters that are central to the ventricular function, such as the stroke volume, ejection fraction, and ventricular mass, have not been determined, and the superiority of this approach relative to $2 \mathrm{D}$ approximation remains not yet critically assessed.

Herein, we report a novel application of pseudodynamic 3D imaging to screen cardioactive and cardiotoxic drugs on zebrafish. We first derive individual parameters (including the end systolic and diastolic volume of a ventricle, stroke volume, ejection fraction, heart rate, ventricular mass, and cardiac output) that underline the cardiac function of zebrafish. Beyond the systolic function, we obtain the ventricular diastolic filling curve, a functional parameter sensitive to cardiac abnormality. These quantities can potentially serve as a baseline for comparison in prospective cardiac pharmacological or translational tests in which zebrafish are employed as a model. As a demonstration for pharmaceutical development, we evaluate both inotropic and chronotropic responses of the heart of zebrafish subject to pharmacological interventions of varied cardiac activities. We reveal also the deranged cardiac function of a zebrafish model of cardiomyopathy. These results show together that the cardiac function of zebrafish exhibits a pharmacological response similar to that of human beings, thereby supporting our approach to serve as a platform to predict the cardiac response of new drugs or chemicals on human beings. We compare also cardiac parameters obtained in this work with those derived with a $2 \mathrm{D}$ approximation (based on the "fit-to-ellipse" algorithm); ${ }^{19}$ we show that the 2D approach tends to overestimate the cardiac parameters and produces results of greater variation. In view of the growing interest of using zebrafish in both fundamental and applied biomedical research, we envisage that our results should benefit fields ranging from exploration of the regenerative mechanism of hearts after injury, ${ }^{20}$ to investigation of the pathophysiology of congenital or acquired heart diseases, ${ }^{21}$ to a pharmaceutical evaluation of cardiac activity and toxicity.

\section{MATERIALS AND METHODS}

Reagents. Phenylthiocarbamide (PTU), epinephrine, doxazosin mesylate, esmolol hydrochloride, tricaine (Sigma Aldrich), and isoflurane (Baxter) were acquired from the indicated sources.

Maintenance and Handling of Zebrafish. Breeding colonies of a transgenic line of zebrafish, $\operatorname{Tg}\left(\mathrm{BMP} 4\right.$ :EGFP), ${ }^{22}$ that expresses enhanced green fluorescence protein (EGFP) in the myocardium were provided by the Taiwan Zebrafish Core Facility and were maintained according to protocols previously reported. $^{23}$ To inhibit the formation of melanophores, PTU was added to the egg medium beginning from one day post fertilization (dpf). The cardiovascular system of zebrafish develops rapidly. By two dpf, the heart tube has differentiated into chambers, showing rhythmic and coordinated contractions; by five dpf, the heart of the embryo has developed with essential configurations similar to the heart of an adult teleost, and a cardiovascular system becomes functional. ${ }^{24,25}$ Throughout this work, zebrafish larvae (6 dpf) exhibiting a regular heartbeat (about 190 beat per min) were used.

Administration of Drugs. Zebrafish larvae were anesthetized with a mixture of tricaine $(100 \mathrm{ppm})$ and isoflurane $(100 \mathrm{ppm}){ }^{26}$ To treat epinephrine $(200 \mathrm{ppm})$ or doxazosin $(50 \mathrm{ppm})$, the indicated drug was injected into the anesthetized larvae through the retro orbital. To administer esmolol (2000 $\mathrm{ppm})$, larvae were immersed in a solution containing the drug and the anesthetic. Doxorubicin $(60 \mathrm{ppm})$ was administrated from five dpf for a duration of $24 \mathrm{~h}$. All measurements were performed $20 \mathrm{~min}$ after administration of the drugs, except the test of doxorubicin in which measurements were made at the end of the treatment, in a room with temperature maintained at $25{ }^{\circ} \mathrm{C}$.

Confocal Laser-Scanning Fluorescent Imaging. We employed a confocal microscope (Leica TCS SP5 II, Leica) equipped with an objective lens $(20 \times / 0.7)$. To produce images, we used a blue laser $(488 \mathrm{~nm}$; typical power below $1 \mathrm{~mW})$ as excitation and detected fluorescence between 510 and $600 \mathrm{~nm}$. Before measurements, we have verified that the larval heart beat periodically with time-lapse $x y$-images obtained at a selected 
imaging plane. To construct 3D images, we recorded $x y$-images $(512 \times 512$ pixels $)$ at a rate of 25 frames per s for a duration of 1 s. The temporal resolution, $40 \mathrm{~ms}$, corresponds to approximately one-seventh of a cardiac cycle. Images at varied depths were obtained sequentially on moving the objective lens at increments of $1 \mu \mathrm{m}$. It took about $90 \mathrm{~s}$ to complete acquisition of images of a larval heart which is about $80 \mu \mathrm{m}$ in the short axis at the diastolic phase.

Reconstruction of Pseudodynamic 3D Images of the Ventricle of a Beating Zebrafish Heart. Pseudodynamic 3D images of a contracting heart were constructed with a protocol modified from the literature. ${ }^{16}$ The principle of image reconstruction is illustrated in a cartoon displayed in Figure 1A. In brief, time-lapse images were first obtained at varied focal depths. As the ventricular wall contracted periodically, the ventricular chamber expanded and shrank during the diastolic and systolic phases of a cardiac cycle (some images acquired at selected depths are displayed in Figure 1B). The image of the largest ventricular chamber in a series was identified and attributed to "end of diastole" (EoD) of the ventricle. Once the image corresponding to EoD was chosen for images in each series, images in two series with adjacent depths were then "synchronized" on aligning those images of EoD. The procedure was applied to images in each series in sequence. This practice allowed registration of each image according to the relative time point of that image in a cardiac cycle. The images aligned in a column were then used to reconstruct a $3 \mathrm{D}$ image, and the resulting 3D image hence corresponded to a snapshot of the ventricle at the registered time point in a cardiac cycle. A 3D image displayed in Figure 1C shows a snapshot of a ventricle, in which the ventricular wall and ventricular chamber are rendered with green and red false colors. All 3D images corresponding to varied time points of a cardiac cycle were then used to construct a video clip to show the contractile motion of the ventricular chamber. Construction of $3 \mathrm{D}$ images and video clips was achieved with software (Imaris, Bitplane).

Determination of Individual Parameters of the Cardiac Function. After construction of 3D images of the ventricle, we calculated the volumes of the ventricular chamber (termed "ventricular volume" hereafter) at each time point of a cardiac cycle with software. Once the temporally varying ventricular volumes were determined, other parameters such as end-diastolic volume (EDV), end-systolic volume (ESV), stroke volume (SV), ejection fraction (EF), heart rate (HR), cardiac output (CO), and ventricular mass (VM) were deduced according to their definitions. ${ }^{27}$ For instance, EDV and ESV correspond to the ventricular volume immediately before a contraction and at the end of the contraction. SV, the volume of blood ejected at a heartbeat, is determined from the difference between EDV and ESV. EF is SV divided by EDV. $\mathrm{CO}$ is $\mathrm{SV}$ multiplied by HR, a quantity depicting the volume of blood propelled in the interval of one minute. Finally, VM is determined with the volume of the ventricular wall multiplied by the density of the ventricular wall $\left(1.05 \mathrm{~g} \mathrm{~cm}^{-3}\right){ }^{28}$

Statistics. Comparison between the means of two groups was made with the two-tailed Student's $t$ test. The levels of statistical significance were set at $* p<0.05$, ** $p<0.01$, and $* * * p<0.001$, respectively.

\section{RESULTS}

Cardiac Function of Zebrafish Larvae at 6 dpf. A result representative of the temporal variation of the ventricular volume of zebrafish larvae at $6 \mathrm{dpf}$ is displayed in Figure 2 (see also a video clip, "CONTROL.avi”, Supporting Information).

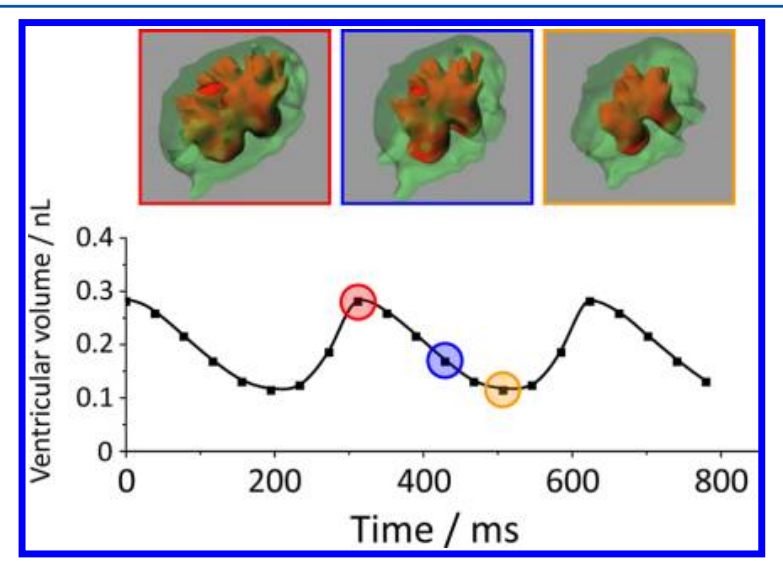

Figure 2. Temporal variation of the ventricular volume determined from an untreated zebrafish larva $(6 \mathrm{dpf})$. The three $3 \mathrm{D}$ images correspond to snapshots of the ventricle obtained at approximately the beginning, middle, and end of the systolic phase of a cardiac cycle.

The three 3D images, displayed in the same figure, correspond to the snapshot of the ventricle caught approximately at the beginning, middle, and end of the systolic period.

To evaluate the variation of the cardiac function among fish, we implemented the same analysis on 15 fish larvae of the same age. The mean and standard deviation of individual parameters are summarized in Table 1 . The result shows that the variation

Table 1. Parameters of the Cardiac Function of Zebrafish Larvae Obtained with Pseudo-Dynamic 3D Imaging $(n=15)$

\begin{tabular}{lc}
\multicolumn{2}{c}{ Cardiac Parameters of Zebrafish Larvae at $6 \mathrm{dpf}$} \\
\hline end-diastolic volume $/ \mathrm{nL}$ & $0.285 \pm 0.050$ \\
end-systolic volume $/ \mathrm{nL}$ & $0.105 \pm 0.025$ \\
stroke volume $/ \mathrm{nL}$ & $0.179 \pm 0.034$ \\
ejection fraction $/ \%$ & $63.1 \pm 5.6$ \\
heart rate $/ \mathrm{min}^{-1}$ & $191.6 \pm 17.1$ \\
cardiac output $/ \mathrm{nL} \cdot \mathrm{min}^{-1}$ & $34.5 \pm 7.4$ \\
ventricular mass $/ \mu \mathrm{g}$ & $0.403 \pm 0.063$ \\
\hline
\end{tabular}

of the cardiac parameters ranged from about $10 \%$ (the ejection fraction and heart rate) to $20 \%$ (the end-diastolic volume, cardiac output, and stroke volume). These values are regarded as the baseline of the normal control for zebrafish larvae at 6 $\mathrm{dpf}$, with which the results determined from the larvae subject to varied interventions were compared.

Pharmacological Effects of Drugs on the Ventricular Systolic Function. To demonstrate an evaluation of the pharmacological response of cardiac function, we used drugs that possess known cardiac activities. Representative results obtained on the treatment of these drugs are displayed in Figure 3. Comparison of the functional parameters between the treated larvae and untreated normal control are summarized in Figure 4.

Epinephrine is an endogenous hormone that exerts both positive inotropic and chronotropic effects on cardiomyocytes and is known to increase both contractility and beating rate of human hearts. Figure $3 \mathrm{~A}$ shows the temporal variation of the ventricular volume of a zebrafish larva after an injection of epinephrine (see also "EPINEPHRINE.avi", Supporting Information); the $3 \mathrm{D}$ snapshots corresponding approximately to 


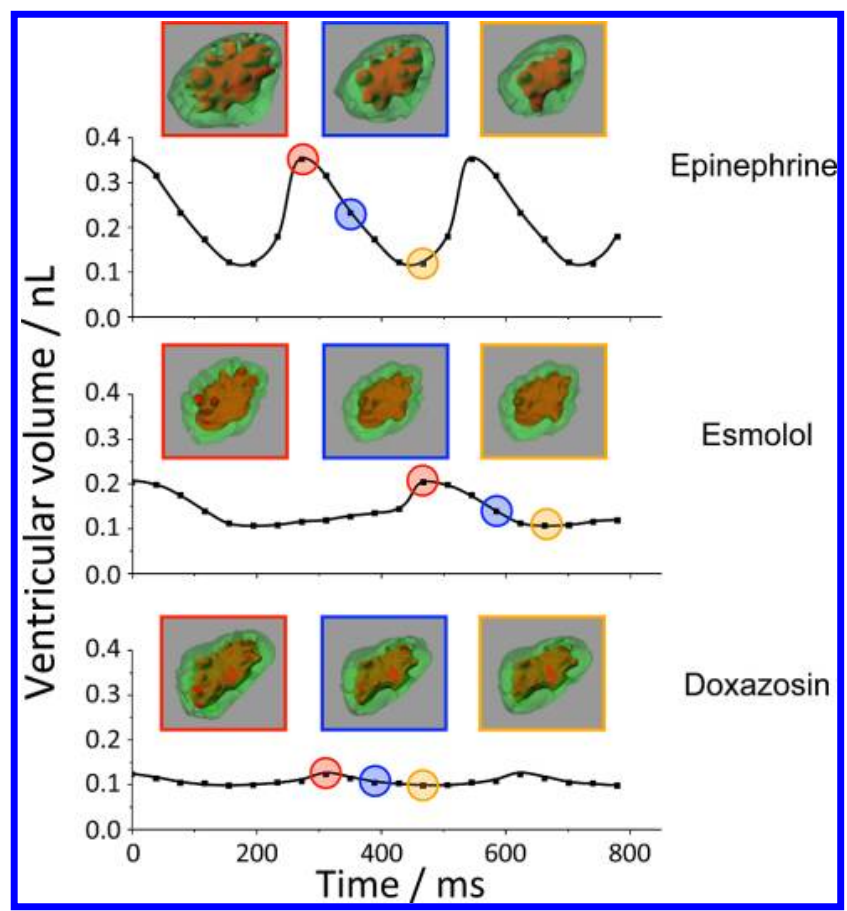

Figure 3. Temporal variation of the ventricular volume of zebrafish treated with epinephrine, esmolol, and doxazosin. The three 3D images displayed on top of each curve are the snapshots of the ventricle obtained at approximately the beginning, middle, and end of the systolic phase of a cardiac cycle.

the beginning, middle, and end of the ventricular systolic phase are also displayed. Compared with the normal control (Figure 2 ), the result shows clearly that epinephrine boosted greatly both the contractility and heart rate of zebrafish larvae. The result displayed in Figure 4 shows further that the administration of epinephrine resulted in a significantly increased stroke volume $(0.232 \pm 0.035 \mathrm{~nL}$ vs $0.179 \pm 0.034$ $\mathrm{nL}, p=0.0027)$, ejection fraction $(69.3 \pm 5.0 \%$ vs $63.1 \pm 5.6 \%$, $p=0.0055)$, heart rate $\left(224 \pm 12 \mathrm{~min}^{-1}\right.$ vs $191 \pm 17 \mathrm{~min}^{-1}, p=$ $0.00013)$, and cardiac output $\left(51.3 \pm 9.4 \mathrm{~nL} \mathrm{~min}^{-1}\right.$ vs $34.5 \pm$ $\left.7.4 \mathrm{~nL} \min ^{-1}, p=0.00025\right)$ relative to the normal control. These observations are profoundly consistent with the known agonistic action of epinephrine on both alpha- and betaadrenergic receptors of cardiomyocytes. In contrast to the substantial increase in the systolic function of the ventricle, there was no discernible change of the ventricular mass after the treatment $(0.406 \pm 0.071 \mu \mathrm{g}$ vs $0.403 \pm 0.063 \mu \mathrm{g}, p=0.86)$, indicating that epinephrine did not cause morphological damage to the ventricular muscle of zebrafish at the dose tested here.

Esmolol is an antagonist of the beta-adrenergic receptor of cardiomyocytes and is known to suppress both inotropy and chronotropy of the heart. As shown in Figure 3B, the heartbeat slowed and the contractility decreased significantly after an administration of esmolol (see also "ESMOLOL.avi", Supporting Information). Conforming to the pharmacological activity of esmolol on human beings, the result displayed in Figure 4 shows that the treatment of esmolol resulted in a significantly decreased stroke volume $(0.076 \pm 0.036 \mathrm{~nL}$ vs $0.179 \pm 0.034$ $\mathrm{nL}, p=0.000032)$, ejection fraction $(39.3 \pm 14.9 \%$ vs $63.1 \pm$ $5.6 \%, p=0.00016)$, heart rate $\left(125 \pm 18 \mathrm{~min}^{-1}\right.$ vs $191 \pm 17$ $\left.\min ^{-1}, p=0.000033\right)$, and cardiac output $\left(9.6 \pm 5.0 \mathrm{~nL} \mathrm{~min}^{-1}\right.$ vs $34.5 \pm 7.4 \mathrm{~nL} \mathrm{~min}^{-1}, p=0.000031$ ) relative to the normal control. Similar to the observations made on a treatment of epinephrine, acute exposure of esmolol had little effect on the ventricular mass $(0.393 \pm 0.039 \mu \mathrm{g}$ vs $0.403 \pm 0.063 \mu \mathrm{g}, p=$ $0.50)$.

To demonstrate selective antagonistic effects, we tested doxazosin mesylate; this drug is an alpha-adrenergic antagonist and is known to suppress only the contractility, not the chronotropic property of human hearts. Consistent with its known selective activity, our results show that the heart rate of

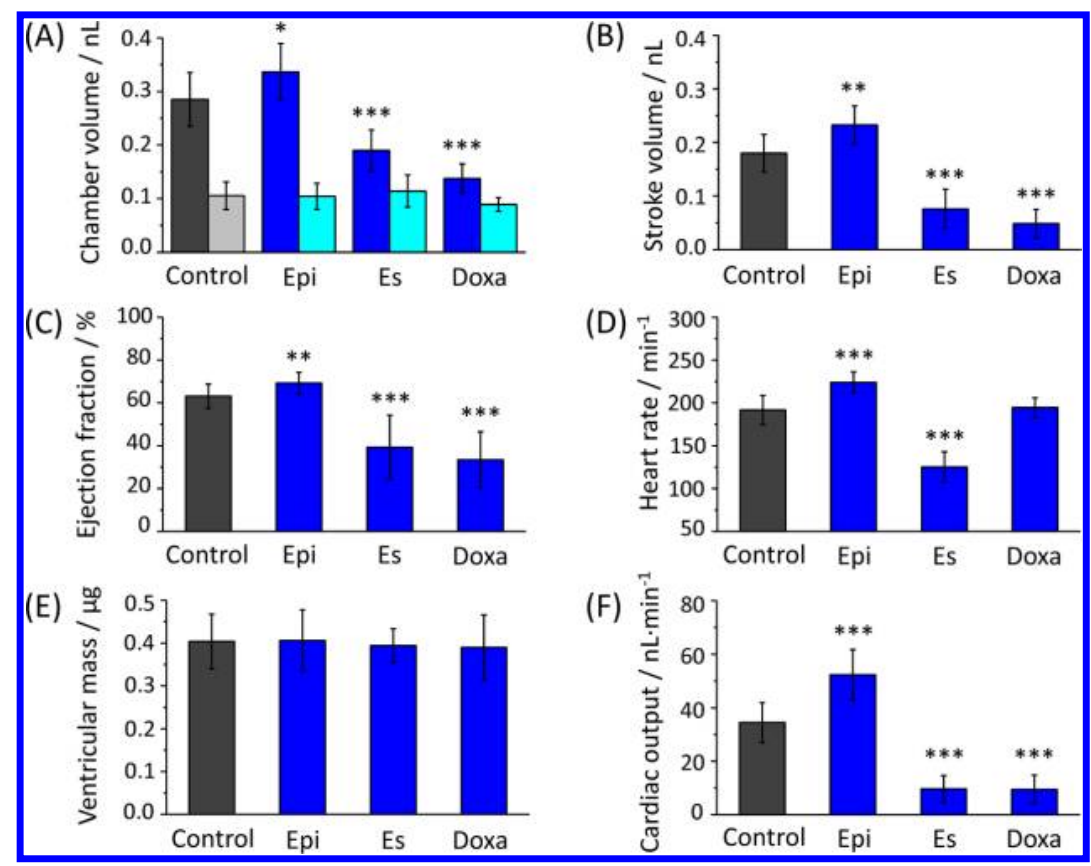

Figure 4. Comparison of the cardiac parameters of untreated zebrafish (control, $n=15$ ) with that treated with epinephrine (Epi, $n=10)$, esmolol (Es, $n=10)$, and doxazosin (Doxa, $n=10)$. (A) End-diastolic and end-systolic volumes of the ventricular chamber; (B) stroke volume; (C) ejection fraction; (D) heart rate; (E) ventricular mass; (F) cardiac output. 


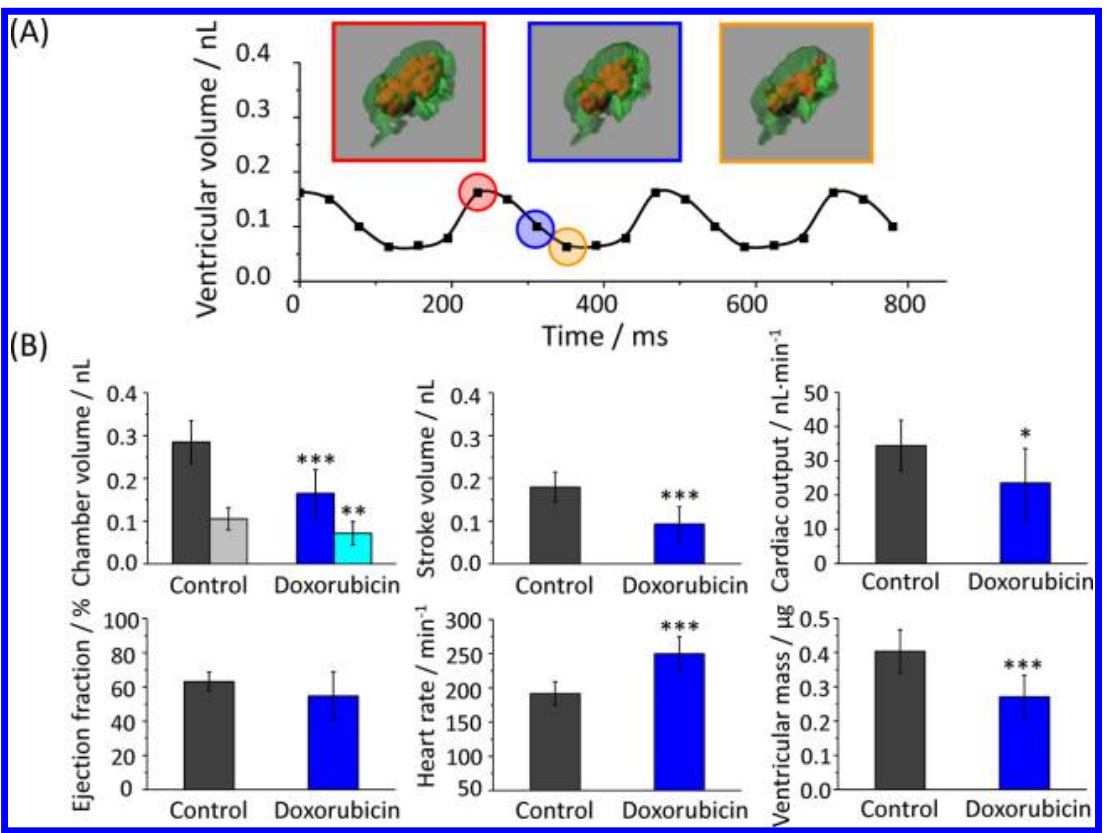

Figure 5. (A) Temporal variation of the ventricular volume of a larva treated with doxorubicin and three snapshots of the ventricle during the systolic phases. (B) Comparison of the cardiac function of zebrafish larvae treated with doxorubicin $(n=10)$ with that of the untreated control $(n=$ $15)$.

zebrafish larvae remained comparable $\left(194 \pm 11 \mathrm{~min}^{-1}\right.$ vs 191 $\left.\pm 17 \mathrm{~min}^{-1}, p=0.92\right)$ whereas the stroke volume $(0.048 \pm$ $0.026 \mathrm{~nL}$ vs $0.179 \pm 0.034 \mathrm{~nL}, p=0.000057)$, ejection fraction $(33.4 \pm 13.1 \%$ vs $63.1 \pm 5.6 \%, p=0.00013)$, and cardiac output

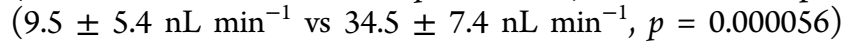
decreased greatly relative to the untreated control (Figures $3 \mathrm{C}$ and 4; see also "DOXAZOSIN.avi", Supporting Information). Similar to the results of epinephrine and esmolol, doxazosin did not alter the ventricular mass of zebrafish larvae $(0.389 \pm 0.076$ $\mu \mathrm{g}$ vs $0.403 \pm 0.063 \mu \mathrm{g}, p=0.32$ ). These results together subtlely demonstrate the selective alpha antagonistic effect, in contrast to that observed on the treatment of esmolol, a betaadrenergic antagonist that suppressed not only the contractility but also the heart rate.

Assessment of Cardiomyopathy Induced by Doxorubicin. Doxorubicin is a chemotherapeutic agent commonly prescribed to patients with hematological malignancies or solid tumors such as breast cancer; it has, however, been shown to be cardiotoxic causing cardiomyopathy of varied severity through an excessive production of reactive oxygen species (ROS). ${ }^{8}$ To explore a potential application of our approach to assess heart diseases of clinical relevance or drugs of cardiotoxicity, we induced cardiomyopathy with doxorubicin and determined the variation of essential cardiac parameters.

As shown in Figure 5, the administration of doxorubicin to zebrafish larvae affected the heart in several respects, causing not only functional impairment but also morphological derangement (see also "DOXORUBICIN.avi", Supporting Information). Specifically, both the stroke volume (0.093 \pm $0.040 \mathrm{~nL}$ vs $0.179 \pm 0.034 \mathrm{~nL}, p=0.00010)$ and the ventricular mass $(0.270 \pm 0.063 \mu \mathrm{g}$ vs $0.403 \pm 0.063 \mu \mathrm{g}, p=0.00017)$ decreased significantly for this zebrafish model of cardiomyopathy relative to the normal control. Although the heart rate increased after treatment with doxorubicin $\left(253 \pm 25 \mathrm{~min}^{-1}\right.$ vs $\left.191 \pm 17 \mathrm{~min}^{-1}, p=0.00031\right)$, the cardiac output decreased significantly $\left(24.1 \pm 10.7 \mathrm{~nL} \mathrm{~min}{ }^{-1}\right.$ vs $34.5 \pm 7.4 \mathrm{~nL} \mathrm{~min}^{-1}, p=$ 0.017 ) because of a remarkable decrease in the stroke volume.
These observations are notably consistent with the decreased contractility and ventricular mass and increased heart rate observed on patients of childhood malignancy who are treated with doxorubicin. ${ }^{29,30}$

Determination of the Diastolic Filling Curve of the Ventricle and Evaluation of the Ventricular Diastolic Function. Diastolic dysfunction typically occurs preceding systolic dysfunction in the progression of human cardiovascular diseases; it is hence of clinical significance to determine the ventricular diastolic function. Diastolic function is typically determined with Doppler echocardiographic measurements on parameters of the trans-mitral flow, such as the early (E) and late (A) diastolic filling rates, ratio $\mathrm{E} / \mathrm{A}$, and the deceleration period or slope of the E-wave in clinical settings. ${ }^{31}$ Despite being a convenient means, the accuracy of diastolic function measured with Doppler echocardiography might be impaired by the preload and afterload of a ventricular chamber. We alternatively determined the diastolic function with the differential temporal variation of the ventricular volume $(\mathrm{d} V /$ $\mathrm{d} t ; V$ denotes the ventricular volume) ${ }^{32}$

To determine the ventricular diastolic function, we first fit the temporal ventricular volume with a polynomial function and differentiated the best fit with respect to time to obtain the diastolic filling curve of the ventricle. A representative ventricular diastolic filling curve of an untreated larva is displayed in Figure 6A. Our result shows that the diastolic filling curve possessed a single maximum within the diastolic phase of a cardiac cycle, with a maximal filling rate of $2.72 \pm$ $0.99 \mathrm{pL} \mathrm{ms}^{-1}$ located at $63 \pm 4 \%$ of the diastolic phase. This result is distinct from what is typically observed on human beings that commonly show two components, an early wave ("E wave") and a late wave ("A wave"), corresponding to ventricular relaxation and atrial contraction, respectively. The observation of a single maximum on zebrafish larvae is attributed to a fusion of the $\mathrm{E}$ and $\mathrm{A}$ waves as a result of the rapid heart rate of zebrafish $\left(191 \pm 17 \mathrm{~min}^{-1}\right)$ relative to human beings (about $70 \mathrm{~min}^{-1}$ ). This attribution is plausible as 


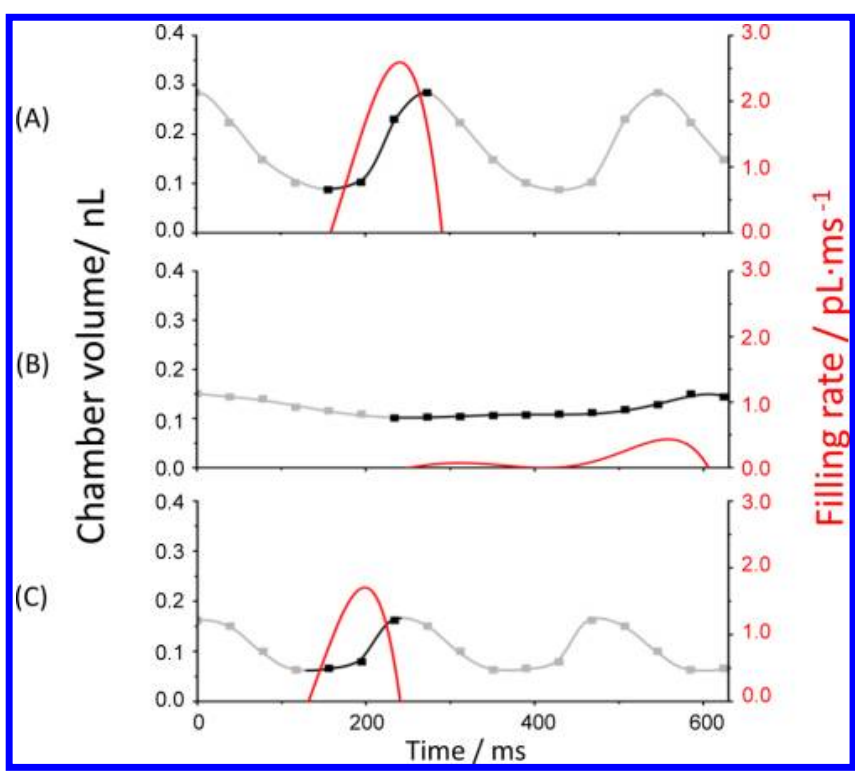

Figure 6. Ventricular diastolic filling curve (red line) derived from the temporal variation of the ventricular volume (gray line). For clarity, the diastolic phase of a cardiac cycle is highlighted with heavy lines. (A) Untreated control; (B) treated with esmolol; (C) treated with doxorubicin.

merging of the $\mathrm{E}$ and A waves is commonly observed also for infants or adults with a high heart rate. ${ }^{31}$

This attribution is supported by the test of esmolol that significantly decreased the heart rate. As shown in Figure 6B, the diastolic filling curve observed on zebrafish treated with esmolol comprised two distinct components: the $\mathrm{E}$ and $\mathrm{A}$ waves located at $17 \pm 6 \%$ and $82 \pm 5 \%$ of the diastolic phase, respectively. In particular, the maximal filling rate of the early wave is much smaller than that of the late wave $(0.17 \pm 0.11 \mathrm{pL}$ $\mathrm{ms}^{-1}$ vs $0.71 \pm 0.67 \mathrm{pL} \mathrm{ms}^{-1}$ ); this result is notably consistent with that reported in adult zebrafish ${ }^{33}$ but distinct from that typically observed for human beings.

Cardiomyopathy induced by doxorubicin has been reported to lead to diastolic dysfunction. Consistently, our result, displayed in Figure 6C, shows that the diastolic filling curve possessed only one maximum with its maximal filling rate significantly smaller than that of the normal control (1.84 \pm $0.74 \mathrm{pL} \mathrm{ms}^{-1}$ vs $2.72 \pm 0.99 \mathrm{pL} \mathrm{ms}^{-1}$ ). As the administration of doxorubicin typically resulted in an increased heart rate, it is hence not surprising that the diastolic filling curve exhibited only one maximum instead of two.

\section{DISCUSSION}

We have evaluated the activity and toxicity of drugs with specific emphasis on their pharmacological effects on the cardiac function. This aspect is of particular significance in view of the prevalence of human cardiovascular diseases and the increasing occurrence of drugs with unforeseen detrimental effects on cardiac function. Our approach can prospectively become an effective platform that facilitates contemporary pharmaceutical development. By determination of the cardiac function of zebrafish models of varied human cardiovascular diseases $^{21,34}$ or wild-type zebrafish, chemicals of desirable cardioprotective effects can be sorted for further testing with higher animals, and drugs of unforeseen cardiotoxicity can be removed before progress to markets. To demonstrate this application, we determined various parameters underlining the ventricular systolic function of zebrafish (Figure 2 and Table 1), tested drugs (epinephrine, esmolol, and doxazosin) of known cardiac activities (Figures 3 and 4), and evaluated the cardiac function of a zebrafish model of cardiomyopathy (Figure 5).

Beyond application in drug screening, our systematic determination of the functional parameters of zebrafish hearts lays the foundation for future tests that require accurate determination of the cardiac function of this important model organism. These results might have widespread impact on research fields ranging from fundamental investigation of the extraordinary regenerative capability of zebrafish hearts after injury, ${ }^{20}$ to a critical evaluation of the therapeutic outcome of novel genetic or stem-cellular interventions that target human heart diseases. ${ }^{35,36}$

In addition to the ventricular systolic function, we determined also the diastolic function of the ventricle (Figure 6A). The diastolic function is particularly sensitive to subtle pathophysiological changes of varied heart diseases, especially at their early stages, ${ }^{37}$ making it a valuable indicator of the healing and recovery of the myocardium after pharmacological or genomic interventions. Our results show a subtle variation of the diastolic filling curve either caused by a pharmacological treatment or associated with a disease model (Figure 6B,C). The ability to determine the diastolic function of zebrafish might facilitate fundamental research on congenital heart diseases using zebrafish with genetic defects. ${ }^{38}$ Our results also call for further efforts to associate varied diastolic filling curves with cardiovascular diseases of specific types. With distinct patterns being identified with specific pathological abnormality, diverse future applications including clinical diagnosis, pharmacological development, or cardiotoxicological screening are expected.

To evaluate critically the superiority of the $3 \mathrm{D}$ volumetric analysis, we compared our results systematically with that obtained with a 2D approximation (termed "fit-to-ellipse"), a method commonly used to determine the systolic function of zebrafish larvae. ${ }^{15,19}$ We first acquired time-course images at a sectional plane near the center of the ventricle and identified the image corresponding to end-diastole. The perimeter of the ventricular chamber was then fitted with an ellipse to determine the major and minor axes. The ventricular volume at enddiastole was calculated according to $V=\left(4 \pi a b^{2}\right) / 3$ in which $a$ and $b$ denote the major and minor axes, respectively. The ventricular volume at end-systole was determined in the same manner.

We analyzed fifteen larvae with this $2 \mathrm{D}$ approximation and $3 \mathrm{D}$ imaging and plotted the ratio between EDV (and ESV) obtained with these two methods for each larva in Figure 7. The result shows clearly that the $2 \mathrm{D}$ approximation tends to produce greater EDV and ESV than with $3 \mathrm{D}$ volumetric analysis. In addition to EDV and ESV, the 2D approximation also overestimates other functional parameters such as SV and CO (data not shown). The $2 \mathrm{D}$ approximation produces notably greater coefficients of variation relative to the 3D approach (28.7\% vs $17.5 \%$ for EDV; $28.9 \%$ vs $23.8 \%$ for ESV). One should hence be cautious when interpreting experimental results based on data obtained with the $2 \mathrm{D}$ approximation. This factor is particularly true when the pharmacological effect of cardioactive agents is evaluated.

In summary, we have demonstrated an accurate evaluation of the cardiac function of zebrafish subject to varied pharmacological interventions and that the $3 \mathrm{D}$ volumetric analysis produces more accurate results than a $2 \mathrm{D}$ approximation. Some 


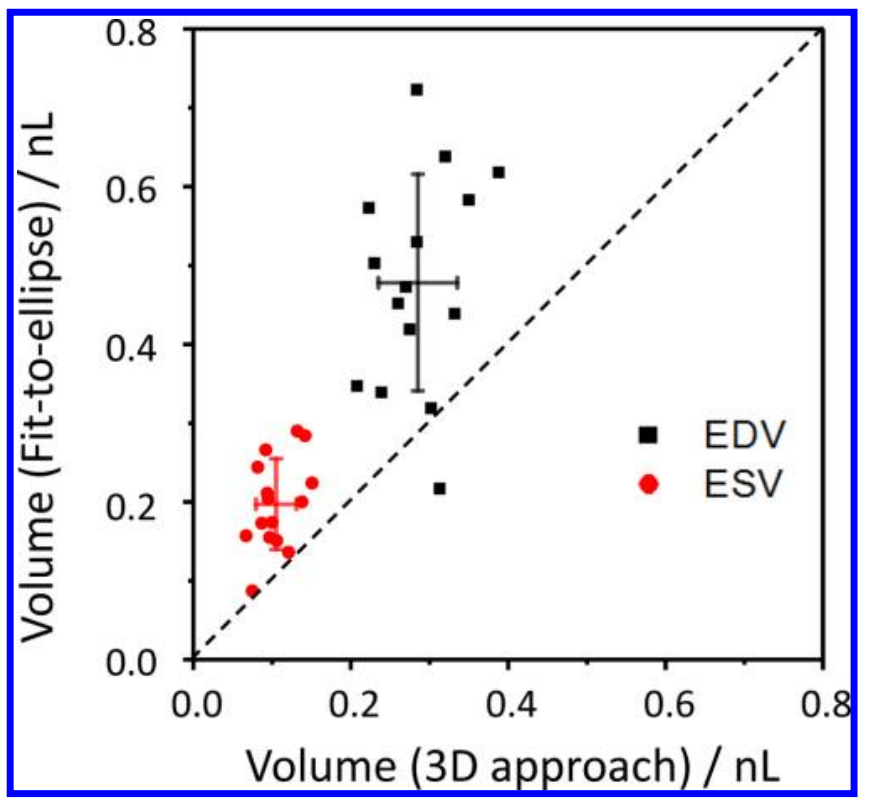

Figure 7. Ratio of ventricular volumes of untreated zebrafish larvae obtained with a $2 \mathrm{D}$ approximation and 3D imaging (black square: enddiastolic volume, EDV; red circle: end-systolic volume, ESV; $n=15$ ). The black and red cross lines indicate the standard deviation of data in each set.

limitations of this approach nevertheless remain. First, pseudodynamic imaging is limited to a determination of the cardiac function of a heart with periodic and regular pulsation, thereby preventing reliable evaluation of the cardiac function of a heart with arrhythmic beating. Second, similar to all optical means, this approach is limited to the depth of penetration of light into tissues. On the basis of our experience, it is difficult to acquire clear images of the heart of zebrafish larvae after $10 \mathrm{dpf}$. This limitation might be eliminated to some extent if twophoton fluorescence imaging ${ }^{39}$ or transgenic fish lines of greater translucency ${ }^{40}$ are employed.

\section{CONCLUSIONS}

In summary, we demonstrate the utility of zebrafish as a novel platform to predict the cardiac activity and cardiotoxicity of drugs on human beings. Our approach can potentially be scaled up becoming an automated analytical platform for highthroughput screening of drugs. Beyond pharmacological development, we anticipate that our approach should benefit also exploration of novel therapeutic strategies that target human heart diseases using zebrafish as disease models.

\section{ASSOCIATED CONTENT}

\section{S Supporting Information}

Supporting video clips as noted in text. This material is available free of charge via the Internet at http://pubs.acs.org.

\section{AUTHOR INFORMATION}

\section{Corresponding Author}

*E-mail: ianliau@mail.nctu.edu.tw.

\section{Author Contributions}

${ }^{\#}$ K.-Y.L. and W.-T.C. contributed equally.

\section{Notes}

The authors declare no competing financial interest.

\section{ACKNOWLEDGMENTS}

We thank Professors Yuan-Pern Lee and Yaw-Kuen Li (National Chiao Tung University) for generous support, and Professor Kwok-Tung Lu (NTNU) for helpful discussion. National Chiao Tung University, National Science Council, and the MOE-ATU program of Taiwan provided support to I.L. We acknowledge the Taiwan Zebrafish Core Facility and grant (\#NSC102-2321-B-001-038) from Taiwan to support the source of zebrafish.

\section{REFERENCES}

(1) MacRae, C. A.; Peterson, R. T. Chem. Biol. 2003, 10, 901-908.

(2) Zon, L. I.; Peterson, R. T. Nat. Rev. Druo Discovery 2005, 4, 3544

(3) Neelkantan, N.; Mikhaylova, A.; Stewart, A. M.; Arnold, R.; Gjeloshi, V.; Kondaveeti, D.; Poudel, M. K.; Kalueff, A. V. ACS Chem. Neurosci. 2013, 4, 1137-1150.

(4) Burns, C. G.; Milan, D. J.; Grande, E. J.; Rottbauer, W.; MacRae, C. A.; Fishman, M. C. Nat. Chem. Biol. 2005, 1, 263-264.

(5) McGrath, P.; Li, C. Q. Drug Discovery Todav 2008, 13, 394-401.

(6) Bowman, T. V.; Zon, L. I. ACS Chem. Biol. 2010, 5, 159-161.

(7) Lloyd-Jones, D.; Adams, R. J.; Brown, T. M.; Carnethon, M.; Dai, S.; De Simone, G.; Ferguson, T. B.; Ford, E.; Furie, K.; Gillespie, C.; Go, A.; Greenlund, K.; Haase, N.; Hailpern, S.; Ho, P. M.; Howard, V.; Kissela, B.; Kittner, S.; Lackland, D.; Lisabeth, L.; Marelli, A.; McDermott, M. M.; Meigs, J.; Mozaffarian, D.; Mussolino, M.; Nichol, G.; Roger, V. L.; Rosamond, W.; Sacco, R.; Sorlie, P.; Stafford, R.; Thom, T.; Wasserthiel-Smoller, S.; Wong, N. D.; Wylie-Rosett, J. Circulation 2010, 121, E46-E215.

(8) Octavia, Y.; Tocchetti, C. G.; Gabrielson, K. L.; Janssens, S.; Crijns, H. J.; Moens, A. L. I. Mol. Cell. Cardiol. 2012, 52, 1213-1225.

(9) Isaacson, C. W.; Usenko, C. Y.; Tanguay, R. L.; Field, J. A. Anal. Chem. 2007, 79, 9091-9097.

(10) Cuello, S.; Ximenez-Embun, P.; Ruppen, I.; Schonthaler, H. B.; Ashman, K.; Madrid, Y.; Luque-Garcia, J. L.; Camara, C. Analyst 2012, 137, 5302-5311.

(11) Chi, N. C.; Shaw, R. M.; Jungblut, B.; Huisken, J.; Ferrer, T.; Arnaout, R.; Scott, I.; Beis, D.; Xiao, T.; Baier, H.; Jan, L. Y.; TristaniFirouzi, M.; Stainier, D. Y. R. PLoS Biol. 2008, 6, 1006-1019.

(12) Basu, S.; Sachidanandan, C. Chem. Rev. 2013, 113, 7952-7980.

(13) Ho, Y. L.; Shau, Y. W.; Tsai, H. J.; Lin, L. C.; Huang, P. J.; Hsieh, F. I. Ultrasound Med. Biol. 2002, 28, 1137-1143.

(14) Dhillon, S. S.; Doro, E.; Magyary, I.; Egginton, S.; Sik, A.; Muller, F. PLoS One 2013, 8, No. e60552.

(15) Chen, Z. Y.; Huang, W.; Dahme, T.; Rottbauer, W.; Ackerman, M. J.; Xu, X. L. Cardiovasc. Res. 2008, 79, 97-108.

(16) Liebling, M.; Forouhar, A. S.; Gharib, M.; Fraser, S. E.; Dickinson, M. E. I. Biomed. Opt. 2005, 10, 054001.

(17) Forouhar, A. S.; Liebling, M.; Hickerson, A.; NasiraeiMoghaddam, A.; Tsai, H. J.; Hove, J. R.; Fraser, S. E.; Dickinson, M. E.; Gharib, M. Science 2006, 312, 751-753.

(18) Liebling, M.; Forouhar, A. S.; Wolleschensky, R.; Zimmermann, B.; Ankerhold, R.; Fraser, S. E.; Gharib, M.; Dickinson, M. E. Dev. Dyn. 2006, 235, 2940-2948.

(19) Kopp, R.; Schwerte, T.; Pelster, B. I. Exp. Biol. 2005, 208, $2123-$ 2134.

(20) Poss, K. D.; Wilson, L. G.; Keating, M. T. Science 2002, 298, 2188-2190.

(21) Chico, T. J. A.; Ingham, P. W.; Crossman, D. C. Trends Cardiovasc. Med. 2008, 18, 150-155.

(22) Shentu, H.; Wen, H. J.; Her, G. M.; Huang, C. J.; Wu, J. L.; Hwang, S. P. L. Genesis 2003, 37, 103-112.

(23) Huang, S. H.; Hsiao, C. D.; Lin, D. S.; Chow, C. Y.; Chang, C. J.; Liau, I. PLoS One 2011, 6, No. e24764.

(24) Stainier, D. Y. R.; Lee, R. K.; Fishman, M. C. Development 1993, $119,31-40$. 
(25) Hu, N.; Sedmera, D.; Yost, H. J.; Clark, E. B. Anat. Rec. 2000, $260,148-157$.

(26) Huang, W. C.; Hsieh, Y. S.; Chen, I. H.; Wang, C. H.; Chang, H. W.; Yang, C. C.; Ku, T. H.; Yeh, S. R.; Chuang, Y. J. Zebrafish 2010, 7, 297-304.

(27) Alfakih, K.; Plein, S.; Thiele, H.; Jones, T.; Ridgway, J. P.; Sivananthan, M. U. I. Magn. Reson. Imaging 2003, 17, 323-329.

(28) Maddahi, J.; Crues, J.; Berman, D. S.; Mericle, J.; Becerra, A.; Garcia, E. V.; Henderson, R.; Bradley, W. L. Am. Coll. Cardiol. 1987, 10, 682-692.

(29) Bulock, F. A.; Mott, M. G.; Oakhill, A.; Martin, R. P. Br. Heart J. 1995, 73, 340-350.

(30) Lipshultz, S. E.; Lipsitz, S. R.; Sallan, S. E.; Dalton, V. M.; Mone, S. M.; Gelber, R. D.; Colan, S. D. I. Clin. Oncol. 2005, 23, 2629-2636.

(31) Nagueh, S. F.; Appleton, C. P.; Gillebert, T. C.; Marino, P. N.; Oh, J. K.; Smiseth, O. A.; Waggoner, A. D.; Flachskampf, F. A.; Pellikka, P. A.; Evangelisa, A. Eur. I. Echocardiogr. 2009, 10, 165-193.

(32) Zeidan, Z.; Erbel, R.; Barkhausen, J.; Hunold, P.; Bartel, T.; Buck, T. I. Am. Soc. Echocardiogr. 2003, 16, 29-37.

(33) Sun, L.; Lien, C. L.; Xu, X. C.; Shung, K. K. Ultrasound Med. Biol. 2008, 34, 31-39.

(34) Arnaout, R.; Ferrer, T.; Huisken, J.; Spitzer, K.; Stainier, D. Y. R.; Tristani-Firouzi, M.; Chi, N. C. Proc. Natl. Acad. Sci. U.S.A. 2007, 104, 11316-11321.

(35) Losordo, D. W.; Vale, P. R.; Symes, J. F.; Dunnington, C. H.; Esakof, D. D.; Maysky, M.; Ashare, A. B.; Lathi, K.; Isner, J. M. Circulation 1998, 98, 2800-2804.

(36) Segers, V. F. M.; Lee, R. T. Nature 2008, 451, 937-942.

(37) Packer, M. Eur. Heart I. 1990, 11, 35-40.

(38) Bakkers, J. Cardiovasc. Res. 2011, 91, 279-288.

(39) Helmchen, F.; Denk, W. Nat. Methods 2005, 2, 932-940.

(40) White, R. M.; Sessa, A.; Burke, C.; Bowman, T.; LeBlanc, J.; Ceol, C.; Bourque, C.; Dovey, M.; Goessling, W.; Burns, C. E.; Zon, L. I. Cell Stem Cell 2008, 2, 183-189. 